priorities. Disease burden can be measured using a variety of indicators such as mortality, morbidity or financial cost. For SLE, despite a relatively good 10 year survival (95\%, vs 97\% in controls), its mortality rate remains high relative to general population mortality rate as shown in our analysis of the Centers for Disease Control (CDC)'s mortality database comprising of 62,843 SLE deaths. However, mortality rates may not adequately measure SLE burden, because among those who died, a fifth of patients died before reaching 40 years of age. Premature mortality, a measure of burden first proposed in 1940 s to address the inadequacy of mortality in measuring the burden of tuberculosis, is an important way to quantify disease burden. In constructing a measure of premature death, an arbitrary limit to life is chosen, and the calculation of the difference between an age at death and this arbitrary limit is defined as the life lost as a result of that death. To assess the relative burden of SLE, we measured the years of potential life lost (YPLL) for SLE relative to CDC's top 15 leading causes of death.

Methods This is a population-based study. Death counts were obtained from the CDC-WONDER database. Number of deaths were tabulated for top 15 CDC's leading causes-ofdeath and SLE. To calculate YPLL, each decedent's age at death from a specific disease was subtracted from a predetermined age of 75 years. The years of potential life lost were then added together to yield the total YPLL.

Results 28411 female SLE deaths were recorded during 2000-2015. The ranking of SLE deaths relative to the CDC's official leading-causes-of-death ranklist in females showed that SLE is within the top 15 leading causes-of-death in reproductive age women (15-44 years) and tenth among women ages 15-24 years. We calculated YPLL for SLE relative to the CDC's official 15 leading causes-of-death in women ages 1544 and 15-24. YPLL for SLE ranked 14th in women ages 15-44, and 8th in women ages 15-24, above diabetes mellitus, HIV disease, septicemia, chronic lower respiratory disease, anemias, nephritis, and cerebrovascular disease.

Conclusions SLE is among the leading cause of YPLL in young women, underscoring SLE as an important public health issue.

\section{CS-33 PERFORMANCE OF URINARY HER2 AS A BIOMARKER OF ACTIVE PROLIFERATIVE LUPUS NEPHRITIS}

${ }^{1}$ Patricia Costa-Reis, ${ }^{2}$ Rebecca E Sadun*, 'Kelly Maurer, ${ }^{2}$ Laura E Schanberg, 'Jon M Burnham, ${ }^{3}$ Emily von Scheven, ${ }^{4}$ Kathleen M O'Neil, ${ }^{5}$ Marisa S Klein-Gitelman, ${ }^{6} \mathrm{M}$ Petri, ${ }^{1}$ Kathleen E Sullivan. ${ }^{1}$ Children's Hospital of Philadelphia, Philadelphia, PA; ${ }^{2}$ Duke University, Durham, NC; ${ }^{3}$ University of California, San Francisco, San Francisco, CA; Indiana University, Indianapolis, IN; ${ }^{5}$ Lurie Children's Hospital, Chicago, IL; ${ }^{6}$ Johns Hopkins University, Baltimore, $M D$

\subsection{6/lupus-2018-Ism.68}

Background Human Epidermal Growth Factor Receptor 2 (HER2) expression is increased in the kidneys of patients with proliferative lupus nephritis (LN) as well as the kidneys of the mouse model NZM2410, though it is not increased in other glomerulonephritides. Preliminary data indicated that urinary HER2 (uHER2) may be elevated in adult patients with proliferative LN (class III and class IV). This study sought to establish whether uHER2 can serve as a biomarker to predict flare of proliferative lupus nephritis in patients with systemic lupus erythematosus (SLE).

Methods We performed a multicenter prospective study of children with $\mathrm{LN}$ as well as a single center prospective study of adults from Johns Hopkins; children and adults without lupus were enrolled as controls. Urine samples were collected at scheduled visits over the course of 4 years. uHER2 was measured by a commercially available ELISA and compared to two other commercially assayable prospective urinary markers of LN, TNF-like weak inducer of apoptosis (TWEAK) and vascular cell adhesion molecule-1 (VCAM-1). Active lupus nephritis was defined as the presence of one or more of the following findings: granular or red blood cell casts, $>5$ red blood cells/high power field with the exclusion of other causes, or proteinuria of $>0.5$ grams/24 hours or recent increase of $>0.5$ grams $/ 24$ hours.

Results One hundred children with LN were enrolled from five academic centers across the United States alongside 91 age-matched controls (mean age 15 years). Children with LN had significantly higher levels of uHER2 compared with controls $(\mathrm{p}<0.01)$; the difference was greater for children with active LN $(\mathrm{p}<0.005)$. uHER2/creatinine correlated with uTWEAK/creatinine $(\mathrm{p}<0.0001)$ and with uVCAM-1/Creatinine $(\mathrm{p}<0.05)$; uHER2 was more sensitive for flare than TWEAK and more specific for flare than VCAM-1. The adult cohort consisted of 142 SLE patients and 23 healthy controls. uHER2/creatinine levels were increased in adult SLE patients when compared to controls $(\mathrm{p}<0.05)$ with the difference also greatest in patients with active LN $(\mathrm{p}<0.005)$.

Conclusion This multicenter prospective study establishes uHER2 as a promising new biomarker for determination of lupus nephritis flare in both children and adults. Combined with implications for HER2 as a driver of mesangial cell proliferation through microRNA downregulation, these results provide further impetus to explore already-available HER2inhibiting drugs, including tyrosine kinases and certain monoclonal antibodies, for the treatment of $\mathrm{LN}$, such as lapatinib (Tyverb) or trastuzumab (Herceptin).

\section{CS-34 INCREASED PREVALENCE OF NOCTURNAL HYPERTENSION AND ATTENUATED BLOOD PRESSURE DIPPING IN PEDIATRIC SLE}

${ }^{1}$ Scott E Wenderfer*, '1 Fallon Campbell, 'Steffy Jose, 'Mario Archila, ${ }^{2}$ Robyn Matloff, ${ }^{2}$ Kari Mynarski, ${ }^{3} \mathrm{D}$ Jack Weaver, ${ }^{4}$ Sarah Hoffman, ${ }^{5}$ Jessica Nelson, ${ }^{5}$ Mahmoud Kallash, ${ }^{6}$ Hermine Brunner, ${ }^{6}$ Mark Mitsnefes. ${ }^{1}$ Baylor College of Medicine, Houston, TX, USA; ${ }^{2} \mathrm{New}$ York Medical College, Valhalla, NY, USA; ${ }^{3}$ Levine Children's Hospital of Carolinas, Charlotte, NC, USA; ${ }^{4}$ Virginia Commonwealth University, Richmond, VA, USA; ${ }^{5}$ The Ohio State University, Columbus, OH, USA; ${ }^{6}$ Cincinnati Children's Hospital, Cincinnati, OH, USA

\subsection{6/lupus-2018-Ism.69}

Background The optimal goals for blood pressure management in SLE are unknown. Uncontrolled blood pressure is a known risk factor for cardiovascular disease. The increased cardiovascular risks associated with SLE are not fully explained by traditional risk factors, such as hypertension and dyslipidemia, at least when based on definitions established for individuals without SLE. One possibility is that the patterns of blood pressure dysregulation in SLE may differ from those in otherwise healthy individuals or patients with obesity/metabolic syndrome.

Methods The BPpSLE study is a cross-sectional multicenter analysis of blood pressure patterns in children with SLE. Inclusion criteria: $\geq 4$ ACR classification criteria for SLE and age $<21$ years. Exclusion criteria: intravenous steroid use within past month, glomerular filtration rate $<60 \mathrm{~mL} / 1.73 \mathrm{~m}^{2}$ / min, or kidney transplant. Interim analysis was performed on 